\title{
A HELLY-TYPE THEOREM ON A SPHERE
}

\author{
M. J. C. BAKER \\ (Received 5 October 1965)
}

\section{Summary}

The purpose of this paper is to prove that if $n+3$, or more, strongly convex sets on an $n$ dimensional sphere are such that each intersection of $n+2$ of them is empty, then the intersection of some $n+1$ of them is empty. (The $n$ dimensional sphere is understood to be the set of points in $n+1$ dimensional Euclidean space satisfying $x_{1}^{2}+x_{2}^{2}+\cdots+x_{n+1}^{2}=1$.)

\section{Introduction}

In Euclidean space a set is called convex if together with any two of its points it contains the entire line segment joining them. The notion of convexity is easily extended to sets in spherical space, that is sets on the $n$ dimensional sphere as defined already: to be convex a set, together with any two of its points, must also contain the minor arc of the great circle joining them.

However, a complication arises if a set contains a pair of points for which this minor arc is not well defined, i.e. a pair of diametrically opposite, or antipodal points. Consequently there are different definitions of spherical convexity according to the conditions laid down for antipodal points, There are four definitions given in Danzer, Grünbaum and Klee [1]: strong convexity, weak, Robinson-, and Horn-convexity. These are in order of increasing generality, and each is implied by the preceding. This paper is concerned only with strong convexity. A set on a sphere is defined to be strongly convex if and only if it does not contain antipodal points, and together with any two of its points contains the minor arc of the great circle joining them.

The following results are used:

THEOREM 1 (Separation) [4]. Two disjoint closed strongly convex sets on a sphere can be strictly separated by a hyperplane through the centre.

THEOREM 2 (Helly) $[1,2]$. If a family of convex sets in $n$ dimensional Euclidean space is such that each intersection of $n+1$ of them is non-empty, then the intersection of them all is non-empty. 
Theorem 3 (Molnár) [3]. If a family of closed strongly convex sets on an $n$ dimensional sphere is such that no $n+2$ of them cover the sphere and each intersection of $n+1$ of them is non-empty, then the intersection of them all is non-empty.

For a survey of separation theorems see Rennie [4], for Helly's theorem and its relatives see Danzer, Grünbaum and Klee [1], who give a comprehensive bibliography of the subject, and for related Helly-type theorems on a sphere using Robinson-convexity see Robinson [5].

The result is first proved for $n+3$ closed sets.

LEMMA. If $n+3$ closed strongly convex sets on an $n$ dimensional sphere are such that each intersection of $n+2$ of them is empty, then the intersection of some $n+1$ of them is empty.

Proof. Let $A, B C, D, \cdots, Z$ be $n+3$ closed strongly convex sets on an $n$ dimensional sphere. Suppose that

(1) each intersection of $n+2$ of them is empty,

(2) no intersection of $n+1$ of them is empty.

We obtain a contradiction.

The sets $B$ and $C \cap D \cap \cdots \cap Z$ are disjoint, so, by the separation theorem they can be strictly separated by a hyperplane through the origin. This hyperplane defines two open hemispheres. Call that containing $B$ the lower hemisphere and the other the upper hemisphere.

Map the upper hemisphere onto the hyperplane parallel to the first hyperplane and tangential to the hemisphere by a projection with vertex the centre of the sphere. It is clear that under such a mapping or its inverse the convexity and intersection properties of sets are preserved. Denote the images under this mapping of those parts of the sets $A, C, D, \cdots, Z$ that lie in the open hemisphere by $A^{\prime}, C^{\prime}, D^{\prime}, \cdots, Z^{\prime}$.

If all the intersections of $n+1$ sets including $A^{\prime}$, and excluding $B^{\prime}$ and one other were non-empty, then, since $C^{\prime} \cap D^{\prime} \cap \cdots \cap Z^{\prime}$ is also nonempty, by Helly's theorem, $A^{\prime} \cap C^{\prime} \cap D^{\prime} \cap \cdots \cap Z^{\prime}$ would be non-empty. This contradicts (1). Therefore at least one of the above intersections must be empty. Suppose, without loss of generality, that $A^{\prime} \cap D^{\prime} \cap \cdots \cap Z^{\prime}$ is empty. Then $A \cap D \cap \cdots \cap Z$ has no point in the upper hemisphere.

Now map the lower hemisphere similarly onto a hyperplane, denoting the image sets by $A^{\prime \prime}, B^{\prime \prime}, C^{\prime \prime}, D^{\prime \prime}, \cdots, Z^{\prime \prime}$.

If $A^{\prime \prime} \cap D^{\prime \prime} \cap \cdots \cap Z^{\prime \prime}$ has a point in this plane, then consider the $n+2$ sets $A^{\prime \prime}, B^{\prime \prime}, D^{\prime \prime}, \cdots, Z^{\prime \prime}$. Every $n+1$ of them have non-empty intersection (2), so, by Helly's theorem, the intersection of them all is 
non-empty. This contradicts (1). Therefore $A^{\prime \prime} \cap D^{\prime \prime} \cap \cdots \cap Z^{\prime \prime}$ is empty, and so $A \cap D \cap \cdots \cap Z$ has no point in the lower hemisphere.

It has now been shown that $A \cap D \cap \cdots \cap Z$ has no point in either of the two open hemispheres. Since it is not empty (2), this intersection must lie entirely in the region of the sphere that separates the two hemispheres. This region is an $n-1$ dimensional sphere.

By Molnár's theorem any $n+2$ of the sets cover the sphere, for otherwise there would be some $n+1$ with empty intersection. Therefore each point on the sphere belongs to at least two of the sets.

There is a point on the $n-1$ dimensional sphere in $A \cap D \cap \cdots \cap Z$. Therefore the antipodal point must belong to $B \cap C$, since no set contains a pair of antipodal points. But $B$ belongs entirely to the lower open hemisphere. Thus a contracdition has been obtained and the lemma established.

\section{4}

The extension to a family of sets whether closed or not is routine. Let $a, b, \cdots, z$ be $n+3$ strongly convex sets on an $n$ dimensional sphere. Consider all the non-empty intersections of $a$ with one or more of $b, \cdots, z$. Choose one point from each of these intersections and denote by $A$ the convex hull of all these points, that is the smallest convex set containing them. Define $B, \cdots, Z$ similarly. Now $A, B, \cdots, Z$ have the same intersection properties as $a, b, \cdots, z$; for if a particular intersection of some of $a, b, \cdots, z$ is non-empty then at least one point of it must belong to the corresponding sets of $A, B, \cdots, Z$ and thus to their intersection. Conversely, if a particular intersection of some of $a, b, \cdots, z$ is empty, then, since $A \subset a, B \subset b, \cdots, Z \subset z$, the corresponding intersection of the sets of $A, B, \cdots, Z$ must be empty. The sets $A, B, \cdots, Z$ are closed and strongly convex, and so by applying the lemma the result for the sets $a, b, \cdots, z$ is obtained.

Finally if the family has more than $n+3$ sets and satisfies the rest of the hypothesis the result follows immediately by considering just $n+3$ of the sets. This completes the proof of the theorem.

I thank Professor B. C. Rennie of the University College of Townsville for interesting me in Helly's theorem.

\section{References}

[1] L. Danzer, B. Grünbaum and V. Klee, 'Helly's theorem and its relatives', in Convexity Proceedings Seventh Symposium in Pure Mathematics, American Mathematical Society (A.M.S. Providence R.I. 1963), Pp. $101-180$.

[2] E. Helly, 'Utber Mengen konvexer Körper mit gemeinschaftlichen Punkten', Jber. Deutsch. Math.-Verein. 32 (1923), 175-176. 
[3] J. Molnár, 'Ủber eine Utbertragung des Hellyschen Satzes in sphärische Räume', Acta Math. Acad. Sci. Hungary, 8 (1957), 315-318.

[4] B. C. Rennie, Unpublished paper.

[5] C. V. Robinson, 'Spherical theorems of Helly type and congruence indices of spherical caps'. Am. J. Math. 64 (1942), 260-272.

R.A.A.F. Academy

Point Cook, Victoria 